\title{
Entwicklung eines impedimetrischen Biosensors für den Nachweis von Antigliadin Autoantikörpern
}

\author{
Thomas Balkenhohl, Falco Beutler, Daniel Schäfer, Fred Lisdat
}

\section{Zusammenfassung}

In der vorliegenden Arbeit wurde ein Biosensor für den Nachweis von Antikörpern gegen Gliadin entwickelt. Gliadine sind Bestandteile der Getreideglutene und verantwortlich für die Manifestation der Zöliakie (Gluten-Unverträglichkeit). Der Biosensor basiert auf der Immobilisierung von Gliadin auf Goldelektroden, die zuvor mit Polystyrensulfonsäure beschichtet worden waren. Die erfolgreiche Immobilisierung wurde mit Hilfe der Quarzmikrowaage dokumentiert. Die Antigen-Antikörper-Bindung konnte durch die Inkubation mit einem Peroxidase-markierten Zweitantikörper und der enzymatischen Oxidation von 3-Amino-9Ethylcarbazol (AEC) verstärkt werden. Die Zunahme in der Elektrodenisolierung durch die Bindungs- und Ablagerungsreaktion konnte durch elektrochemische Impedanzspektroskopie (EIS) in Anwesenheit des Hexacyanoferrat-Redoxsystems gemessen werden. Die Spektren wurden mit Hilfe eines Randles-Ersatzschaltbildes ausgewertet. Hierbei konnte eine Zunahme im Ladungstransferwiderstand festgestellt werden, die proportional zur Antigliadin-Antikörperkonzentration, im Bereich von $10^{-8} \mathrm{M}$ bis $10^{-6} \mathrm{M}$, war. Mit Hilfe dieses Sensors wurden schließlich humane Seren hinsichtlich ihrer Konzentration an Gliadinantikörpern, sowohl für Immunglobuline vom Typ IgG als auch IgA, untersucht.

\begin{abstract}
In this study an immunosensor was developed for the analysis of anti-gliadin antibodies. These antibodies can serve as an early diagnostic marker for celiac disease. The sensor is based on polystyrenesulfonic acid modified gold electrodes on which gliadin was immobilized. The anti-gliadin antibody recognition was amplified by a second binding step with a peroxidase-labeled antibody and subsequent peroxidase-catalyzed oxidation of 3-amino-9-ethylcarbazole (AEC) resulting in a precipitate formation on the electrode. The change of the electrode surface properties was followed by impedance spectroscopy in the presence of ferri-/ferrocyanide. By evaluating the impedance spectra the charge transfer resistance was found to be a suitable sensor parameter. A calibration curve for the detection of anti-gliadin antibodies was established for antibody concentrations between $10^{-8} \mathrm{M}$ and $10^{-6}$ $\mathrm{M}$ and the sensor was also applied for the analysis of serum samples.
\end{abstract}

\section{Einführung}

Die Autoimmunerkrankung Zöliakie wird durch den Verzehr von Gluten ausgelöst. Gluten ist wichtiger Bestandteil von Getreide, wie Weizen, Gerste und Roggen, und ermöglicht erst die Verwendung dieser Getreidesorten zum Brotbacken. Bei Personen, die an einer Zöliakie leiden, führt der Verzehr von glutenhaltigen Nahrungsmitteln zu einer allergischen Reaktion im Dünndarm. Für die Auslösung dieser Reaktion sind hauptsächlich Gliadine verantwortlich. Gliadine sind schwerlösliche Proteine, mit Molekulargewichten zwischen $30 \mathrm{kDa}$ und $70 \mathrm{kDa}$ [1]. Es kommt zu einer Immunreaktion, in deren Verlauf Antikörper gegen Gliadine, aber auch gegen körpereigene Proteine wie Gewebstransglutaminase gebildet werden. Die Bildung dieser beiden Antikörper führt schließlich zu einer schweren Schädigung des Dünndarms, welche die Nahrungsverwertung im Verdauungstrakt erheblich beeinträchtigt.
Die hohe Spezifität der Antikörper-Antigen Interaktion bildet auch die Basis für einen frühen Nachweis dieser Krankheit. Diagnostische Tests untersuchen hierbei das Serum des Patienten auf die Anwesenheit von Autoantikörpern gegen Gliadin und Gewebstransglutaminase.

Für eine ganze Reihe von klinischen und nichtklinischen Anwendungen steht die Entwicklung solcher immunologischer Testverfahren im Fokus der aktuellen Forschung [2-5]. Immunsensoren werden bereits in der klinischen Diagnostik [6], dem Umweltmonitoring [7-8] und der Lebensmittelanalytik [9] eingesetzt. Für die Entwicklung von tragbaren diagnostischen Analysegeräten, die vor Ort eingesetzt werden können, bietet hierbei die elektrochemische Auslese der Testverfahren eine vielversprechende Alternative zu herkömmlichen optischen Techniken [10-12]. Hierbei werden die Targetmoleküle (Antigene oder Antikörper) auf Elektrodenoberflächen immobilisiert [13]. Dies kann durch einfache Absorption [14], durch Knüpfung von kovalenten Bindungen 
[15] oder durch Einschluss in Polymere [16] geschehen. Wie in herkömmlichen Immunoassays, so wird auch hier das durch den Analyten ausgelöste Signal durch einen zweiten Enzym-markierten Antikörper verstärkt. Die Quantifizierung des Produkts der enzymatischen Reaktion kann schließlich durch amperometrische oder potentiometrische Messungen erfolgen [17-18].

Unter den vielfältigen zur Verfügung stehenden elektrochemischen Messtechniken ist die elektrochemische Impedanzspektroskopie (EIS) eine effektive Methode, um Volumen- wie auch Grenzflächeneigenschaften zu analysieren. Insbesondere können damit Veränderungen an Elektrodenoberflächen detektiert werden. Hierdurch kann auch ein besseres Verständnis der physikalischchemischen Prozesse, welche an Veränderungen von Oberflächenbeschichtungen beteiligt sind, erlangt werden. Die Impedanzspektroskopie ist eine sehr sensitive Methode, bei der man den komplexen Wechselstromwiderstand eines Systems bestimmt, indem man den Wechselstrom in Abhängigkeit einer Wechselspannung bei unterschiedlichen Frequenzen misst [19]. Die EIS findet beispielsweise Anwendung in der Charakterisierung von Membranen auf Sensoroberflächen [20], dem Nachweis von Antigen-Antikörper-Interaktionen [21-22] und der Detektion von Nukleinsäure-Hybridisierungen [23].

Für die Immobilisierung des Targetmoleküls, sei es nun der Antikörper oder das Antigen, gibt es verschiedene Möglichkeiten. Ein Beispiel ist die Verwendung von selbstorganisierenden Thiolmonoschichten (SAMs) [24-25]. Im Fall von langkettigen Thiolen kann hier die Antikörper-Antigen Bindung durch die Änderung in der elektrischen Kapazität nachgewiesen werden [25-26]. Im Gegensatz dazu erlaubt die Immobilisierung auf kurzkettigen SAMs, wie Cystamin oder Mercaptopropionsäure [27-28], einen Nachweis der Bindung durch Widerstandsmessungen. Ein neuer Ansatz für die Immobilisierung von Molekülen nutzt den Ladungsüberschuss von Polyelektrolyten. Hierbei werden die Elektrodenoberflächen mit Hilfe eines Polyelektrolytfilms modifiziert, so dass Biomoleküle durch elektrostatische Bindung an geladene funktionelle Gruppen des Polyelektrolyten an die Elektrodenoberfläche gebunden werden [29-31]. Neben den elektrostatischen Wechselwirkungen tragen hier vermutlich auch Wasserstoffbrückenbindungen und insbesondere hydrophobe Wechselwirkungen zu Interaktionen bei [32-33].

In dieser Arbeit sollte zunächst die Bindungskapazität verschiedener Oberflächenmodifizierungen für Gliadin durch QCM-Analysen untersucht werden. Basierend auf einer geeigneten Elektrodenmodifizierung, wurde dann ein Biosensor für die Detektion und Quantifizierung von Antigliadin-Autoantikörpern entwickelt. Die Auslese des Sensorsignals erfolgte durch elektrochemische Impedanzspektroskopie.

\section{Experimenteller Teil}

\subsection{Materialien}

Weizengliadin, Antigliadin-Antikörper aus Kaninchen, Peroxidase-konjugierte Anti-Kaninchen-Antikörper, 3-Amino-9-Ethylcarbazol (AEC), Chromogen Kit, Polyanilinsulfonsäure, Polystyrensulfonsäure, 3-Mercaptopropionsäure, Propanthiol, Ethanthiol, 2-Mercaptoethanol, N-(3-Dimethylaminopropyl)-N'-Ethyl-Carbodiimid (EDC), N-Hydroxysuccinimid (NHS), Rinderserumalbumin (BSA), Borsäure, Trichloressigsäure, Dinatriumhydrogenphosphat und Natriumdihydrogenphosphat wurden von Sigma-Aldrich (Taufkirchen) bezogen. Peroxidase-konjugierte Antikörper gegen humane Immunglobuline vom Typ IgG und IgA wurden von Seramun Diagnostica GmbH (Wolzig) zur Verfügung gestellt. 2-Morpholinoethansulfonsäure (MES) und 4-(2-Hydroxyethyl)-1-Piperazin-1-Ethansulfonsäure (HEPES) wurden bei AppliChem (Darmstadt); Eisessig, Ethanol, Kaliumhydroxid und Tween20 wurden von Roth (Karlsruhe) bezogen. Quarzkristalle (7.995 MHz) mit einer aktiven Flächen von $0.205 \mathrm{~cm}^{2}$ stammen von der Firma ICM in Oklahoma City (USA). Golddrahtelektroden kamen von Goodfellow (Bad Nauheim).

\subsection{Puffer und Lösungen}

Boratpuffer wurde durch Einstellung des pH-Wertes einer Borsäurelösung mit Hilfe von KOH hergestellt. Die Einstellung des $\mathrm{pH}$-Wertes der Phosphatpuffer erfolgte durch Mischen von äquimolaren Lösungen aus Hydrogenphosphat und Dihydrogenphosphat bis der gewünschte $\mathrm{pH}$ Wert erreicht war. Puffer 1:50 mM Borat, pH 5.5 wurde für die Präparation des Gliadins genutzt. Puffer 2:5 mM Phosphat, pH 7.0 wurde für die QCM-Analysen und die Aufnahme von Impedanzspektren gebraucht. Puffer 3: $10 \mathrm{mM}$ Phosphat, $137 \mathrm{mM}$ Natriumchlorid, 0,05\% (v/v) Tween20, pH 7.0 fand Verwendung in den verschiedenen Inkubationsschritten des Immunoassays. Für die Präparation des Gliadins kamen zwei verschiedene Methoden zur Anwendung: Methode A: $50 \mathrm{mg}$ Gliadin wurde in einer Lösung bestehend aus $500 \mu \mathrm{l}$ Essigsäure und $500 \mu \mathrm{ll}$ Ethanol, gelöst und anschließend mit Puffer 1 auf 2,5 ml aufgefüllt. Diese Lösung wurde dann mit Hilfe einer Sephadex PD-10 Säule (Amersham Biosciences) filtriert. Für die Equilibrierung und Eluierung der Säule wurde Puffer 1 verwendet. Die Bestimmung der Proteinkonzentration des Eluates erfolgte nach Bradford. Diese Prozedur der Gliadinpräparation wurde für die QCM-Analysen und den Aufbau von Immunsensoren auf Polyelektrolyt-modifizierten Elektrodenoberflächen verwendet. Methode B: 3 g Gliadin wurde über Nacht und leichtem Schütteln in einer Lösung bestehend aus 40\% 50 mM MES pH 4,5 und $60 \%$ Ethanol (v/v), gelöst. Nach einer anschließenden Zentrifugation (8000 g, 15 min) wurde das Pellet verworfen und der Überstand mit Trichloressigsäure (Endkonzentration: $10 \%(\mathrm{w} / \mathrm{v})$ ) versetzt. Die Fällung der Proteine erfolgte über $2 \mathrm{~h}$ bei $4^{\circ} \mathrm{C}$. Die gefällten Proteine wurde durch eine Zentrifugation (8000 g, 15 min) 

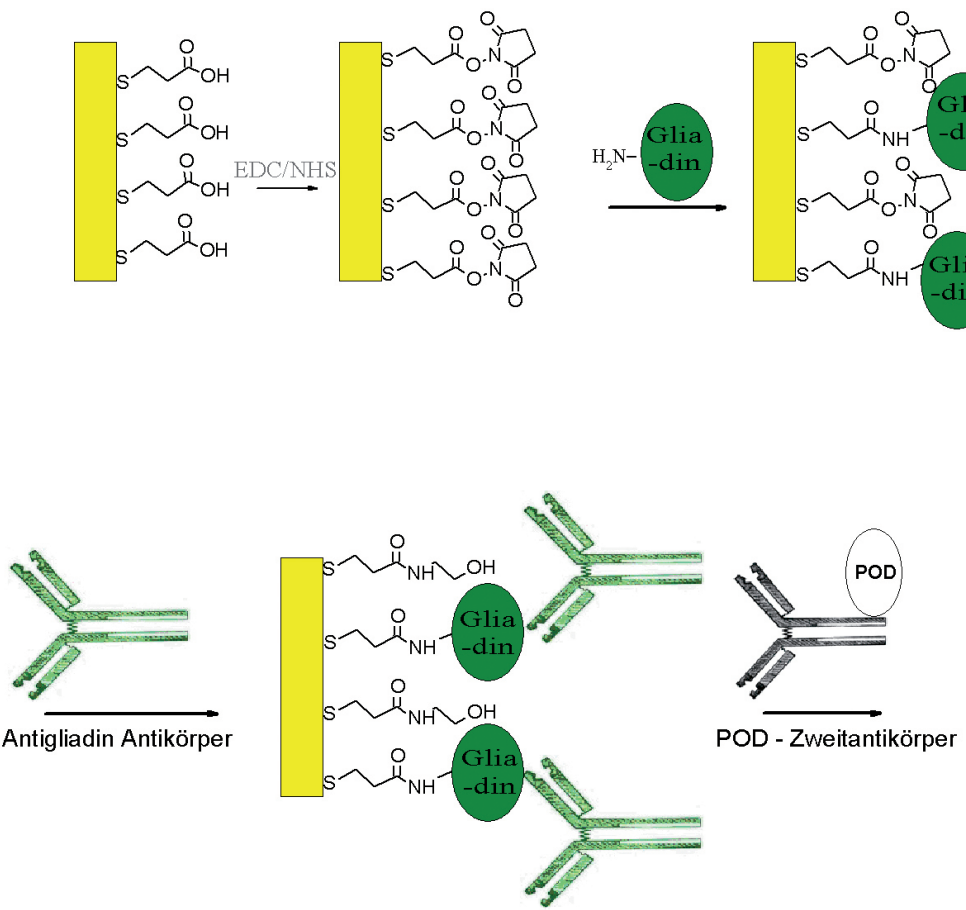
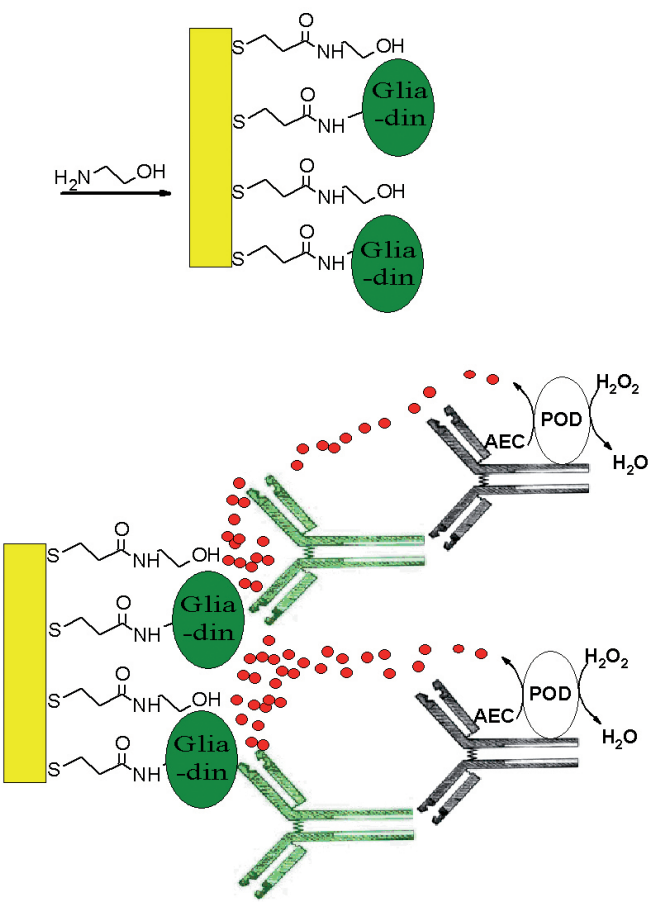

Schema 1: Schematische Darstellung der Gliadin-Immobilisierung und der Testprozedur einer mit einem SAM aus 3-Mercaptopropionsäure modifizierten Goldelektrode. Nach Aktivierung der Carboxylgruppen durch EDC/NHS erfolgt eine Kopplung der Proteine über ihre Aminogruppen. Noch vorhandene aktivierte Carboxylgruppen werden durch Zugabe von Aminoethanol geblockt. Eine Signalverstärkung der Antigen-AntikörperBindung wird durch einen POD-markierten Zweitantikörper ermöglicht. Hierbei katalysiert die Peroxidase die Oxidation von AEC, was zu einem Präzipitat auf der Elektrodenoberfläche führt.

präzipitiert und in einer Mischung aus $40 \%$ HEPES pH 7.0 und $60 \%$ Ethanol (v/v) gelöst. Der Lösungsprozess dauerte $10 \mathrm{~h}$ und wurde durch leichtes Schütteln unterstützt. Nach der Entfernung von ungelösten Bestandteilen durch einen kurzen Zentrifugationsschritt (8000 g, 3 min) wurde die Proteinkonzentration des Überstandes (Gliadin) nach Bradford [35] bestimmt. Diese GliadinPräparation wurde für den Aufbau von Immunsensoren auf 3-Mercaptopropionsäure-modifizierten Elektrodenoberflächen verwendet.

\subsection{Messungen mit der Quarzmikrowaage}

QCM Messungen wurden an einem CHI 400 (IJ Cambria Scientific Ltd, UK) durchgeführt. Für die Messungen im Durchfluss wurde eine selbstgebaute Durchflusszelle mit einem Volumen von ca. $30 \mu \mathrm{l}$ verwendet (Abb. 1). Vor der Oberflächenmodifizierung der goldbeschichteten Schwingquarze wurde diese für $10 \mathrm{~min}$ mit PiranhaLösung (konz. Schwefelsäure, $30 \% \mathrm{H}_{2} \mathrm{O}_{2} ; 3: 1$ ) gereinigt, mit destilliertem Wasser abgespült und anschließend in Batchzellen montiert. Die Goldoberflächen der Schwingquarze wurden hier durch die Inkubation mit verschiedenen Thiolen bzw. Polyelektrolyten für $12 \mathrm{~h}$ modifiziert. Die verwendeten Lösungen sind in Tab. 1 zusammengefasst. Schwingquarze, nach der SAM-Modifizierung, wurden vor der QCM-Analyse mit einer EDC/ NHS-haltigen Lösung inkubiert (je 100 mM, 30 min), um die Carboxylgruppen der 3-MA zu aktivieren. Anschließend wurden diese mit Wasser abgespült, aus der Batchzelle genommen und in die Flusszelle eingebaut. Die QCM-Messungen wurden im Durchfluss bei einer Flussrate von $50 \mu \mathrm{l} / \mathrm{min}$ durchgeführt. Hierbei wurden zunächst die Schwingquarze mit Puffer 2 gespült, bis eine stabile Basislinie erreicht wurde, um anschließend den Puffer durch eine Gliadinlösung $(0,31 \mathrm{mg} / \mathrm{ml}$ in Puffer 1$)$ zu ersetzen. Nachdem $500 \mu \mathrm{l}$ der Gliadinlösung über die Schwingquarzoberfläche geflossen waren, wurde wieder zu Puffer 2 gewechselt.

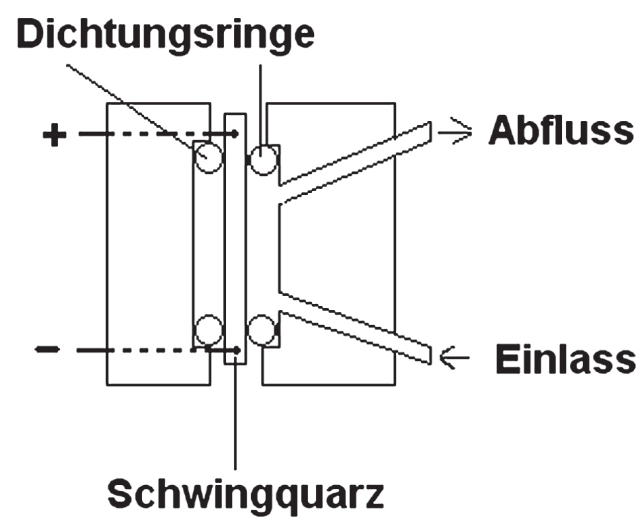

Abb. 1: Schematische Darstellung der QCM Fließzelle.

\subsection{Oberflächenmodifikation der Goldelektro- den und Durchführung des Immunoassays}

Um eine geeignete Oberflächenmodifizierung der Elektroden für die Immobilisierung von Gliadin herzustellen, wurden zwei verschiedene Ansätze verfolgt:

1) (Schema 1) Die Goldelektrode wurde für $12 \mathrm{~h}$ mit einer 10-prozentigen Mercaptopropionsäurelösung (in destilliertem Wasser) inkubiert, um eine homogene SAM zu erhalten. Nach einem kurzen Wasch- 


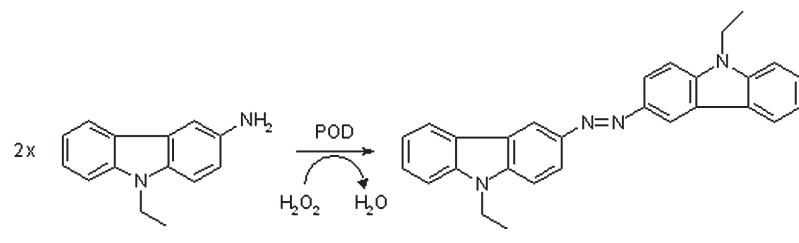

3-Amino-9-Ethylcarbazol

3-Azo-9-Ethylcarbazol

Schema 2: Peroxidase katalysierte Oxidation von 3-Amino-9-Ethylcarbazol (AEC) zu 3-Azo-9-Ethylcarbazol. Das Oxidationsprodukt fällt auf der Elektrodenoberfläche aus und führt so zu einer Isolierung der Elektrode.

schritt wurden die Carboxylgruppen, wie unter 2.3 beschrieben, durch EDC/NHS aktiviert, die Aktivierungslösung entfernt und die Elektrode schließlich für $4 \mathrm{~h}$ mit einer Gliadinlösung $(0,29 \mathrm{mg} / \mathrm{ml})$ inkubiert. Anschließend erfolgte ein Blockierungsschritt, bei dem verbliebene aktivierte Carboxylgruppen mit Hilfe von Aminoethanol (10\% in destilliertem Wasser; $1 \mathrm{~h}$ ) abgesättigt wurden. Die so hergestellten Biosensoren konnten für den Nachweis von Gliadinantikörpern verwendet werden. Hierzu wurden diese mit humanen Seren oder mit Verdünnungen einer Standardlösung eines käuflichen Gliadinantikörpers (aus Kaninchen) für $1.5 \mathrm{~h}$ inkubiert. Nach Waschen der Elektroden in Puffer 3 folgte eine weitere Inkubation mit einem POD-konjugierten Zweit-Antikörper gegen Kaninchen-Immunglobuline (Standardantikörper) oder gegen humane Immunglobuline (Seren) für $1 \mathrm{~h}$. Die Konzentration des POD-Konjugats betrug $1.2 \cdot 10^{-7} \mathrm{M}$ (in Puffer 3 ). Nach einem letzten Waschschritt in Puffer 3 wurden die Biosensoren in einer AEC-Lösung unter leichtem Schütteln für 10 min inkubiert. Hierbei wird AEC durch die Peroxidase zu 3-Azo-9-Ethylcarbazol oxydiert, dies fällt auf der Elek- trodenoberfläche aus (Schema 2). Nach 10 min wurde die AEC-Oxidation durch Umsetzen der Biosensoren in destilliertem Wasser gestoppt und diese schließlich durch EIS analysiert.

2) Die zweite Variante der Modifizierung von Goldelektroden ist in Schema 3 dargestellt: Hier wurden die Elektroden über Nacht mit einer $1 \mathrm{mg} / \mathrm{ml}$ PSS-Lösung versetzt, anschließend mit destilliertem Wasser gewaschen und in Gliadinlösung für $12 \mathrm{~h}$ inkubiert. Es kam zu elektrostatischen Bindungen zwischen positiv geladenen Gruppen des Gliadins und dem negativ geladenen Polyelektrolyten. Nach Entfernung der Gliadinlösung erfolgte ein Blockierungsschritt mit Rinderserumalbumin ( $2 \mathrm{mg} / \mathrm{ml}$ in Puffer $3 ; 1 \mathrm{~h}$ ). Anschließend wurden humane Seren oder Verdünnungen des Standard-Antigliadinantikörpers analysiert. Die Detektion der Antikörper wurde wie oben beschrieben durchgeführt.

\subsection{Elektrochemische Impedanzspektroskopie}

Die Impedanzmessungen wurden mit Hilfe eines IM6e (Zahner, Kronach) in einer konventionellen Drei-Elektroden-Anordnung durchgeführt. Als Referenzelektrode diente eine $\mathrm{Ag} / \mathrm{AgCl} / 1 \mathrm{M} \mathrm{KCl-Elektrode} \mathrm{(Biometra,}$ Göttingen). Das Volumen der Messzelle betrug $1 \mathrm{ml}$. Die Impedanzmessungen wurden in Puffer 2 bei einem Potenzial von $0 \mathrm{~V}$ durchgeführt. Messungen mit $2,5 \mathrm{mM}\left[\mathrm{Fe}(\mathrm{CN})_{6}\right]^{3-}$ und $2,5 \mathrm{mM}\left[\mathrm{Fe}(\mathrm{CN})_{6}\right]^{4-}$ erfolgten bei einem Potential von 0,2 V. Der Frequenzbereich reichte von $500 \mathrm{mHz}$ bis $50 \mathrm{kHz}$. Die Amplitude des Wechselspannungspotenzials betrug $10 \mathrm{mV}$. Die gemessenen Impedanzwerte wurden mit Hilfe der Thales-Software (Zahner) anhand eines passenden Schaltbildes ausgewertet.
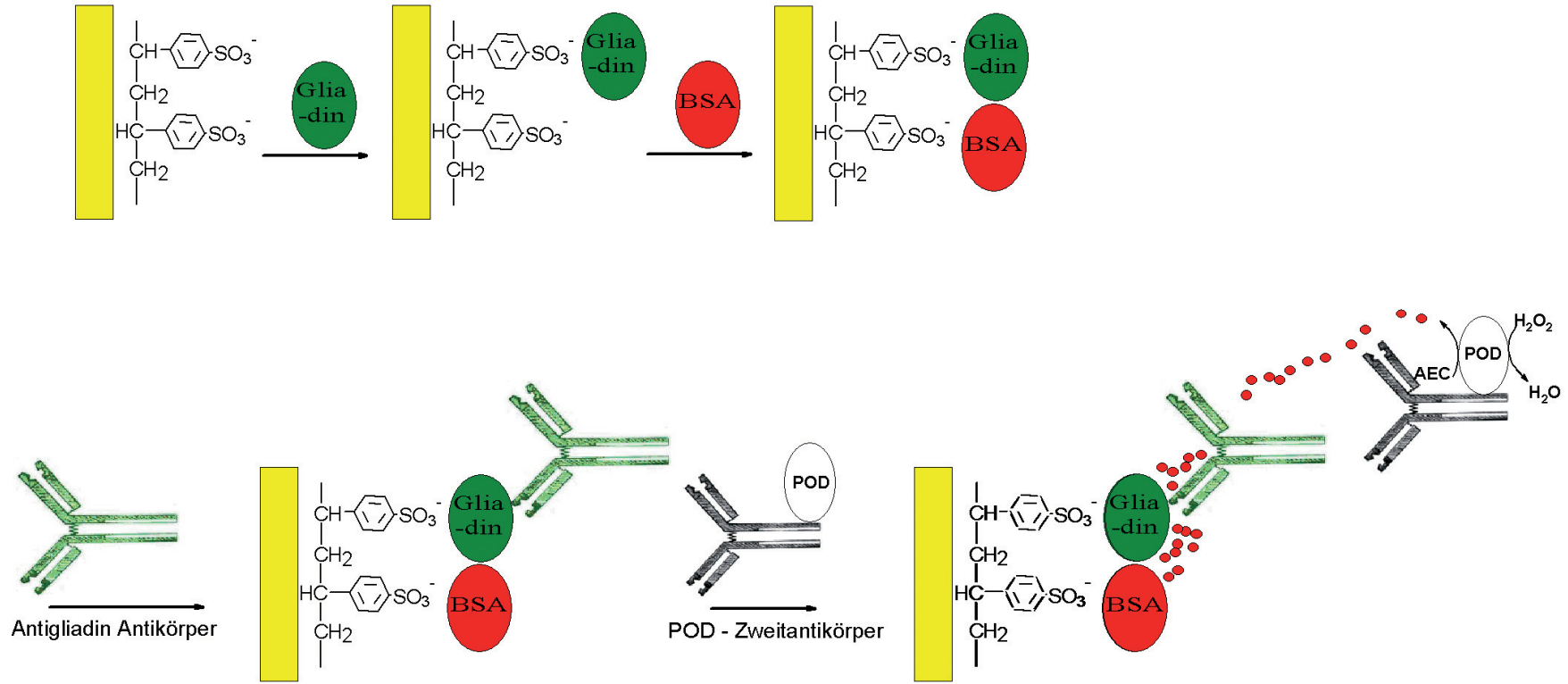

Schema 3: Schematische Darstellung der Gliadin-Immobilisierung, Testprozedur und Signalverstärkung einer PSS-modifizierten Goldelektrode. Die Bindung der Proteine erfolgt über elektrostatische Wechselwirkungen mit dem negativ geladenen Polyelektrolyten. Anschließend werden restliche Bindungsstellen mit Rinderserumalbumin (BSA) abgesättigt. Eine Signalverstärkung der Antikörper-Antigen-Bindung erfolgte wie in Schema 1 beschrieben. 


\section{Ergebnisse und Diskussion}

3.1 Analyse verschiedener Oberflächenmodifizierungen hinsichtlich ihrer Bindungskapazität für Gliadin mit Hilfe der QCM

Um verschiedene Oberflächenmodifizierungen hinsichtlich ihrer Bindungskapazität für Gliadin zu untersuchen, ist die Quarzmikrowaage eine geeignete Methode. Für die in Tab. 1 aufgeführten SAMs und Polyelektrolyten wurde die Bindungskapazität ermittelt. In Abb. 2 sind die Frequenzänderungen der Schwingquarze mit den einzelnen Oberflächenmodifizierungen nach Applikation der Gliadin-Lösung dargestellt. Es wird deutlich, dass drei verschiedene Oberflächenmodifizierungen in der Lage waren, Gliadin zu binden. Am effektivsten war der Polyelektrolyt PSS. Hier konnte eine Verringerung der Frequenz von $\Delta \mathrm{f}=95 \mathrm{~Hz}$ gemessen werden. Der Polyelektrolyt PASA zeigte eine geringere Kapazität für Gliadin (Frequenzabnahme $\Delta \mathrm{f}=45 \mathrm{~Hz}$ ), was möglicherweise an der geringeren Ladungsdichte des Polyelektrolyten lag. Eine Oberflächenmodifizierung mit SAMs aus 3-Mercaptopropionsäure zeigte ebenfalls eine deutliche Bindung von Gliadin. Die Frequenzabnahme des Schwingquarzes betrug $\Delta \mathrm{f}=71 \mathrm{~Hz}$. Im Gegensatz dazu ergaben SAMOberflächen aus Mischungen verschiedener Thiole keine signifikante Bindung.

\begin{tabular}{|l|l|l|l|l|l|}
\hline Variation & $3-\mathrm{MA}$ & PSH & ESH & PSS & PASA \\
\hline a & $10 \%$ & & & & \\
\hline b & $10 \%$ & $10 \%$ & & & \\
\hline c & $10 \%$ & & $10 \%$ & & \\
\hline d & & & & $1 \mathrm{mg} / \mathrm{ml}$ & \\
\hline e & & & & & $1 \mathrm{mg} / \mathrm{ml}$ \\
\hline
\end{tabular}

Tab. 1: Verschiedene Lösungen für die Modifizierung der Goldoberfläche von Schwingquarzen, um anschließend darauf Gliadin zu binden. Alle Lösungen wurden mit destilliertem Wasser hergestellt, Prozentangaben beziehen sich auf das Volumen. Abkürzungen: 3-MA (3-Mercaptopropionsäure), PSH (Propanthiol), ESH (Ethanthiol), PSS (Polystyrensulfonsäure), PASA (Polyanilinsulfonsäure).

Nimmt man für Gliadin ein mittleres Molekulargewicht von $50 \mathrm{kDa}$ an, so kann mit Hilfe der Sauerbrey-Gleichung [36] für die PSS-Schicht eine Oberflächenbelegung von ca. $13 \mathrm{pmol} / \mathrm{cm}^{2}$ berechnet werden. Hierbei muss jedoch berücksichtigt werden, dass die Sauerbrey-Gleichung keine Gültigkeit für flüssige bzw. hydratisierte Verbindungen, wie gelöste Proteine, besitzt, so dass dieser Wert nur als Anhaltspunkt dienen kann. Trotz dieser Einschränkung hinsichtlich der quantitativen Werte ist die QCM aber eine geeignete Methode, um die verschiedenen Oberflächenmodifikationen in Bezug auf ihre relative Bindungskapazität vergleichend zu untersuchen [37-38].

\subsection{Nachweis von Antigliadin-Antikörpern durch Impedanzspektroskopie}

Nach der Immobilisierung der Gliadine auf Goldstabelektroden und anschließender Blockierung wurden diese als Biosensoren für den Nachweis und die Quantifizierung der Antikörper eingesetzt. Die Antikörper-Anti-

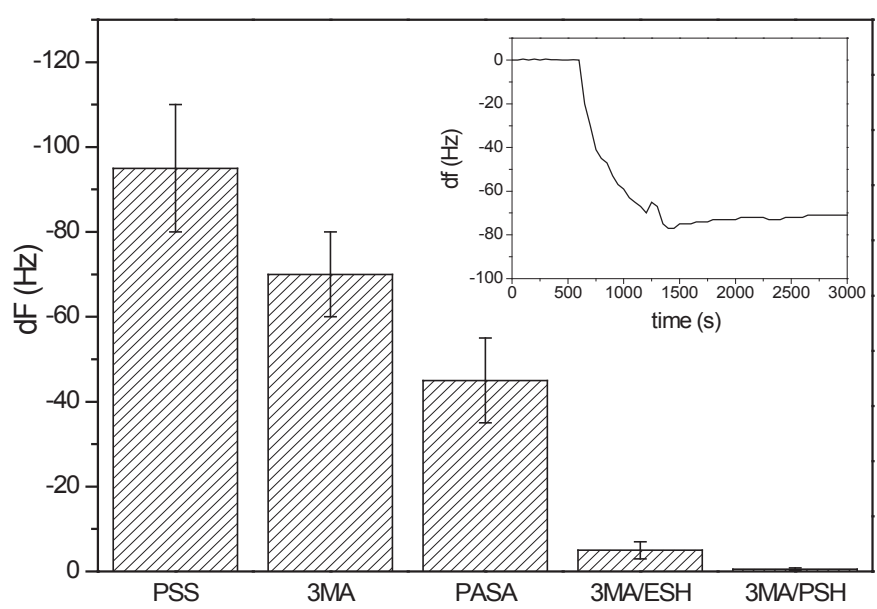

Abb 2: QCM-Analysen der Bindungskapazität für Gliadin an verschieden modifizierten Goldoberflächen. Die Messungen wurden in einer selbstgebauten Fließzelle mit einem Volumen von ca. $30 \mu l$ durchgeführt (Abb. 1). Nachdem der in die Zelle eingebaute Schwingquarz mit Puffer 2 bis zur Einstellung einer Basislinie gespült worden war (Flussrate: $50 \mathrm{\mu l} / \mathrm{min}$ ), wurde der Puffer durch eine Gliadinlösung $(0.31 \mathrm{mg} / \mathrm{ml})$ ersetzt. Nach der Applikation von ca. $500 \mu \mathrm{l}$ der Gliadinlösung bei einer Flussrate von $50 \mu \mathrm{l} / \mathrm{min}$ wurde wieder zu Puffer 2 gewechselt. Jeder Wert repräsentiert den Durchschnitt von zwei analysierten Schwingquarzen mit der gleichen Oberflächenmodifizierung. Beispielhaft zeigt das Inset die QCMKurve eines 3-MA-modifizierten Schwingquarzes.

gen-Bindung und die Peroxidase-katalysierte Oxidation von AEC resultiert in einer Änderung des isolierenden Charakters der Oberflächenmodifizierung der Elektrode. Diese Änderung kann durch EIS quantifiziert werden. Nach den einzelnen Inkubationsschritten wurden Impedanzspektren in Anwesenheit des Redoxsystems $\left[\mathrm{Fe}(\mathrm{CN})_{6}\right]^{3-/ 4-}$ aufgenommen. Abb. 3 zeigt diese Spektren für einen Biosensor auf PSS-Basis. Diese weisen für

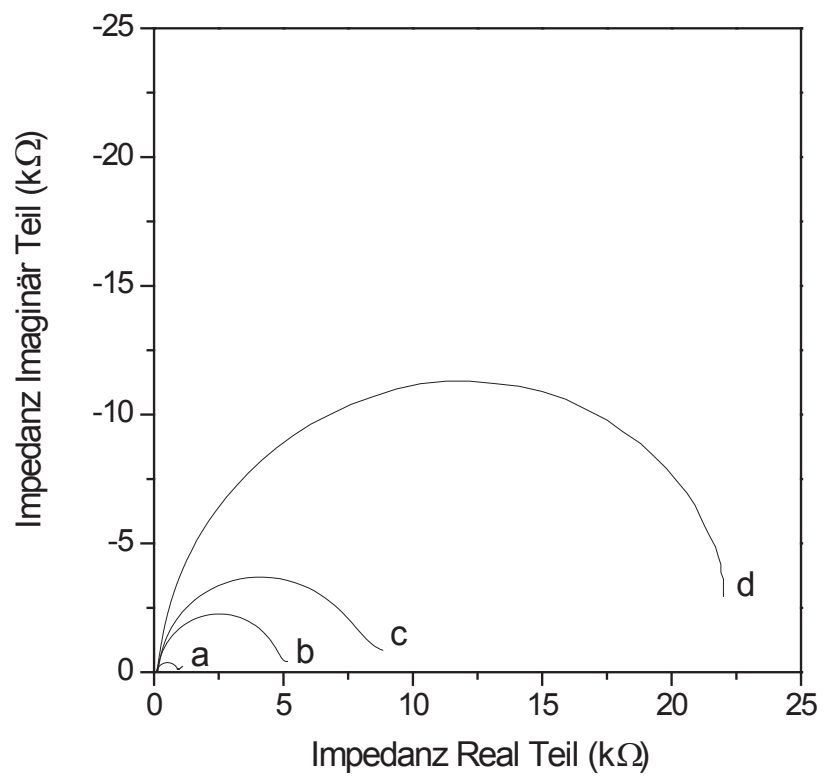

Abb. 3: Nyquist-Darstellung der Impedanzspektren nach den verschiedenen Inkubationsschritten der Testprozedur. Die Messkurven wurden in Puffer 2 in Anwesenheit von $2.5 \mathrm{mM}\left[\mathrm{Fe}(\mathrm{CN})_{6}\right]^{3-}$ und $2.5 \mathrm{mM}\left[\mathrm{Fe}(\mathrm{CN})_{6}\right]^{4-}$ innerhalb eines Frequenzbereichs von $0.5 \mathrm{~Hz}$ bis $50 \mathrm{kHz}$ aufgenommen: (a) PSS modifizierte Elektrode nach GliadinBindung und BSA-Blockierung, (b) Biosensor nach der Inkubation mit Antigliadin-Antikörpern, (c) Biosensor nach der Inkubation mit dem POD-markierten Zweitantikörper, (d) Biosensor nach Oxidation von AEC durch die Peroxidase. 
R/C-Parallelschaltkreise typische Halbkreise auf, welche den Elektronentransferprozess des Hexacyanoferrats an der Elektrodenoberfläche widerspiegeln. Hierbei sind der Elektronentransferwiderstand $\left(\mathrm{R}_{\mathrm{ct}}\right)$ und die Doppelschichtkapazität $\left(\mathrm{C}_{\mathrm{dl}}\right)$ die Elemente, welche die Gestalt der Halbkreise bestimmen. Aus der Abbildung wird deutlich, dass die Inkubation des Biosensors mit dem Antigliadin-Antikörper zu einer Widerstandszunahme führt. Die Bindung des POD-markierten Zweitantikörpers bringt eine weitere, wenn auch kleine Erhöhung. Eine sehr große Widerstandszunahme konnte dagegen nach der POD-katalysierten Oxidation von AEC und Niederschlagsbildung beobachtet werden. Biosensoren auf Basis eines SAMs aus 3-Mercaptopropionsäure zeigten ebenfalls eine Zunahme der Impedanz im Verlauf des Assays (nicht gezeigt). Im Vergleich zum PSS-Biosensor war die Widerstandszunahme jedoch etwas geringer. Um die Impedanzanalyse der Biosensoren zu vereinfachen, wurde auch versucht, Messungen in Abwesenheit eines redoxaktiven Stoffes durchzuführen (nur im Phosphatpuffer). Beispielhaft dafür sind die Impedanzspektren eines Biosensors nach den verschiedenen Inkubationsschritten in Abb. 4 dargestellt. Im Vergleich zu den Messungen mit Redoxmediator resultieren aus der Analyse der Elektroden wesentlich höheren Impedanzwerte. Diese waren ebenfalls nach der AEC-Oxidation am höchsten (Linie c), jedoch war die relative Zunahme im Verlauf des Assays wesentlich geringer. In diesen Experimenten musste auch festgestellt werden, dass eine Zunahme der Impedanz in Kontrollexperimenten (Biosensoren, die nur mit dem POD-markierten Zweitantikörper und nicht mit dem Antigliadin-Antikörper behandelt worden waren) zu messen war. Aus diesen Gründen wurde bei weiteren Impedanzanalysen in Gegenwart des HexacyanoferratRedoxsystems gemessen. Dieses Messschema sorgte für stabile und reproduzierbare Ergebnisse mit maximaler Signaländerung.

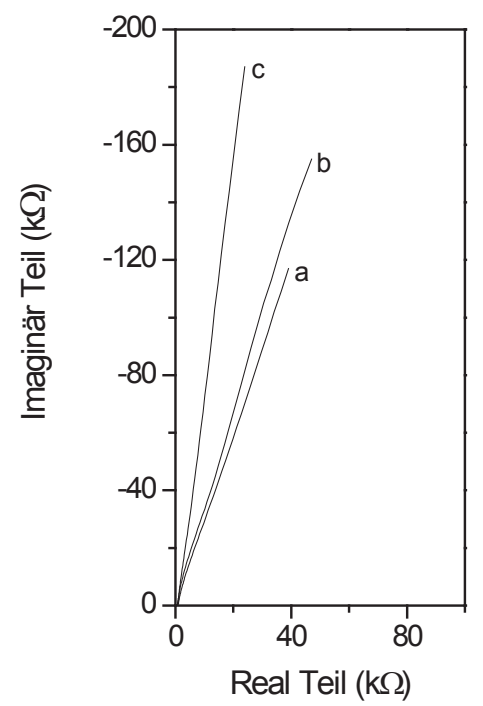

Abb. 4: Impedanzspektren eines auf 3-MA-Basis hergestellten Biosensors nach Messung in reinem Phosphatpuffer ohne Zugabe eines Redoxmediators: (a) 3-MA-modifizierte Elektrode nach Gliadin-Bindung und Aminoethanol-Blockierung, (b) Biosensor nach Inkubation mit dem POD-markierten Zweitantikörper, (c) Biosensor nach Oxidation von AEC durch die Peroxidase.

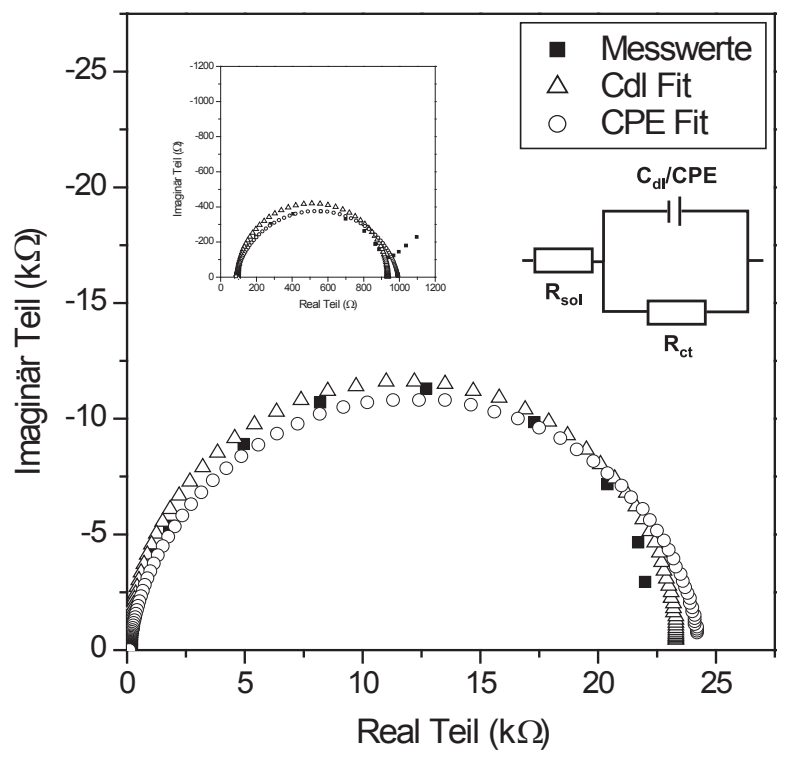

Abb. 5: Aufgenommene Impedanzspektren (-) und anhand eines Randles-Ersatzschaltbildes gefittete Kurven eines PSS-basierten Biosensors nach der BSA-Blockierung (Inset) und nach AECPräzipitation. ( $\triangle$ ) Werte anhand des Fits des Ersatzschaltbildes mit Doppelschichtkapazität $\left(C_{d l}\right)$, (०) Werte anhand des Fits des Ersatzschaltbildes mit konstantem Phasenelement (CPE). Die Fit-Ergebnisse der Schaltbildelemente des Biosensors nach der BSA-Blockierung waren folgende: Ersatzschaltbild mit $C_{d l}$-Element; $R_{\text {sol }}=95 \Omega, R_{c t}=840 \Omega, C_{d l}=1.31 \mu \mathrm{F}$ ) und Ersatzschaltbild mit CPE-Element; $R_{\text {sol }}=88 \Omega, R_{c t}=908 \Omega, C P E=1.19 \mu \mathrm{F}, \alpha=0.88$. FitErgebnisse der Schaltbildelemente des Biosensors nach Präzipitation: Ersatzschaltbild mit $C_{d l^{\prime}}$ Element; $R_{\text {sol }}=10 \Omega, R_{c t}=23.25 \mathrm{k} \Omega, C_{d l}=$ $1.31 \mu \mathrm{F}$; Ersatzschaltbild mit CPE-Element; $R_{\text {sol }}=103 \Omega, R_{c t}=24.21 \mathrm{k} \Omega$, $C P E=1.08 \mu F, \alpha=0.93$.

\subsection{Auswertung der Impedanzspektren anhand eines Ersatzschaltbildes}

Impedanzspektren werden mit Hilfe von Ersatzschaltbildern quantitativ ausgewertet. Ein einfaches und sehr häufig verwendetes Modell ist der Randles-Schaltkreis [39]. Dieser setzt sich aus den Elementen für den Lösungsmittelwiderstand $\left(\mathrm{R}_{\mathrm{sol}}\right)$, der Doppelschichtkapazität $\left(\mathrm{C}_{\mathrm{dl}}\right)$ und dem Ladungstransferwiderstand $\left(\mathrm{R}_{\mathrm{ct}}\right)$ zusammen (Abb. 5). Mit Hilfe des Randles Ersatzschaltbildes wurden das Spektrum des unbenutzten Biosensors (Inset in Abb. 5) und des Biosensors nach absolviertem Assay (Abb. 5) analysiert. Alternativ zur Doppelschichtkapazität wurde in den Randles-Schaltkreis ein konstantes Phasenelement (CPE) integriert. Abb. 5 macht deutlich, dass beide Verfahren eine zuverlässige Kurvenapproximation des Spektrums zulassen und ähnliche Werte für den Ladungstransferwiderstand berechnen. Für den unbenutzten Biosensor ergibt das Randles-Ersatzschaltbild mit Doppelschichtkapazität einen $\mathrm{R}_{\mathrm{ct}}$-Wert von $840 \Omega$ während mit CPE $908 \Omega$ berechnet werden. Nach der Assay-Prozedur wurden 23,2 k $\Omega$ für das $\mathrm{R}_{\mathrm{ct}}$-Element im Ersatzschaltbild mit $\mathrm{C}_{\mathrm{dl}}$-Element und 24,2 k $\Omega$ für $\mathrm{R}_{\mathrm{ct}}$ im Ersatzschaltbild mit dem CPE-Element errechnet. Weiterhin konnte festgestellt werden, dass die kapazitiven Werte in beiden Ersatzschaltbildern $\left(\mathrm{C}_{\mathrm{dl}}: 1.31 \mu \mathrm{F} /\right.$ CPE: $1.19 \mu \mathrm{F}$ (unbenutzter Biosensor) und $\mathrm{C}_{\mathrm{dl}}: 1.31 \mu \mathrm{F} /$ CPE: $1.08 \mu \mathrm{F}$ (Biosensor nach dem Assay) sich nur wenig unterscheiden. Dies wird auch durch den $\alpha$-Faktor des CPE-Elements deutlich, der einen Wert nahe 1 besitzt 
(0.88 für den unbenutzten Biosensor und 0.93 für den Biosensor nach Assay-Prozedur) und somit für das CPEElement eine nur geringfügige Abweichung gegenüber einer reinen Kapazität aufzeigt. Es wird also deutlich, dass die Verwendung eines CPE-Elements im RandlesErsatzschaltbild keine signifikante Verbesserung bietet. Daher wurde für die weitere Auswertung der Impedanzspektren ein Ersatzschaltbild mit $\mathrm{C}_{\mathrm{dl}}$-Element verwendet. Bei dem Vergleich der Werte für die einzelnen Elemente vor dem Assay und danach konnte festgestellt werden, dass die kapazitiven Änderungen des Biosensors durch die Assay-Prozedur nur gering sind (1 - 5\%) und der Lösungsmittelwiderstand (wie erwartet) nicht durch die Antigen-Antikörper-Bindung beeinflusst wird. Es konnte aber eine drastische Zunahme im Ladungstransferwiderstand festgestellt werde, so dass der Wert für das $\mathrm{R}_{\mathrm{ct}}{ }^{-}$ Element als Sensorparameter genutzt werden konnte. Die Auswertung der Impedanzspektren eines Biosensors auf Basis einer SAM mit 3-Mercaptopropionsäure zeigte ein sehr ähnliches Ergebnis und bestätigte, dass eine zuverlässige Auswertung der Spektren anhand eines RandlesErsatzschaltbildes mit einem $\mathrm{C}_{\mathrm{dl}}$-Element möglich ist.

\subsection{Kalibrierung des Biosensors}

Die Verwendung von Goldelektroden als Biosensoren für die Analytik von Antikörpern sollte durch die Etablierung einer Kalibrationskurve und durch die Analyse verschiedener humaner Seren gezeigt werden. Aufgrund der einfachen und schnellen Präparation wurden hierzu Biosensoren auf Basis von PSS-modifizierten Goldelektroden verwendet. Eine Konzentrationsabhängigkeit des Sensorsignals von der Antikörperkonzentration konnte durch die Inkubation der Sensoren in Lösungen mit verschiedenen Konzentrationen an Antigliadin-Antikörpern demonstriert werden. Abb. 6 zeigt eine Kalibrationskurve, in der die $\mathrm{R}_{\mathrm{ct}}$-Werte nach POD-Reaktion gegen den Logarithmus der Antikörper-Konzentration aufgetragen wurden. Es wird deutlich, dass bei Antikörperkonzentrationen im Bereich von $0,008 \mu \mathrm{M}$ bis $1,5 \mu \mathrm{M}$ eine Zunahme im Ladungstransferwiderstand festgestellt werden kann. Eine sigmoidale Kurvenapproximation nach Gleichung 1 [40] resultierte in einer halbmaximalen Sättigung des Sensorsignals bei 0.613 pM. Die Kalibrationskurve weist eine Korrelation mit den Messwerten von $r^{2}=0.96$ auf. Das Detektionslimit des Biosensors kann mit ca. $100 \mathrm{nM}$ angegeben werden.

$$
y=A 2+\frac{A 1-A 2}{1+\left(\frac{x}{x_{0}}\right)^{p}}
$$

$\mathrm{A} 2=$ maximaler $\mathrm{R}_{\mathrm{ct}}$-Wert $\left(1.64 \times 10^{6} \mathrm{Ohm}\right)$

$\mathrm{A} 1=$ minimaler $\mathrm{R}_{\mathrm{ct}}$-Wert $\left(0.25 \times 10^{6} \mathrm{Ohm}\right)$

$\mathrm{X}=$ Konzentration an Antigliadin Antikörpern in der Probe $(\mathrm{M})$

$\mathrm{x}_{0}=$ Halbsättigung des Biosensors mit Antikörpern $(0.613 \mu \mathrm{M})$

$\mathrm{y}=$ Sensorparameter $\left(\mathrm{R}_{\mathrm{ct}}\right.$-Wert $)$ der Probe $(\mathrm{Ohm})$

$\mathrm{p}=$ Exponent (1.19)

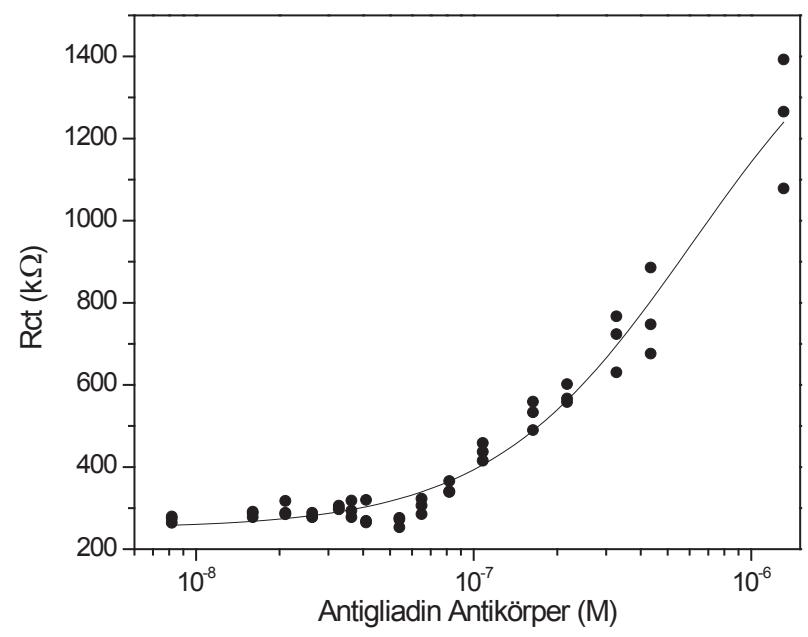

Abb. 6: Kalibrationskurve für Antigliadin-Antikörper: Auftragung des Ladungstransferwiderstandes nach der AEC-Präzipitation gegen den Logarithmus der Antikörperkonzentration (IgG). Jeder Datenpunkt entspricht dem Mittelwert der Messwerte von drei Biosensoren.

Schließlich wurden fünf verschiedene humane Seren, die hinsichtlich ihrer Antigliadin-Antikörper-Konzentration durch ELISA-Experimente eindeutig charakterisiert worden waren (Seramun Diagnostica GmbH, Wolzig), mit Hilfe des Biosensors analysiert. Von den fünf untersuchten Seren waren zwei positiv auf Zöliakie (P1 und P2) und drei Seren negativ (N1 bis N3). Um zuverlässige diagnostische Ergebnisse zu bekommen, wurden diese Seren sowohl auf Antigliadin-Antikörper vom Immunglobulin Typ G als auch Typ A untersucht. Dies wird möglich durch die Verwendung unterschiedlicher PODmarkierter Antikörper; einerseits gegen humane IgGs und andererseits gegen IgAs. Obwohl die Analytik von IgG-Molekülen einfacher und sensitiver ist, reicht eine alleinige Bestimmung von IgG-Antigliadin-Antikörpern nicht aus, da diese auch in anderen, ähnlichen Autoimmunerkrankungen vorkommen und daher ein positiver Befund nicht zwingend für Zöliakie wäre. Antikörper vom Typ IgA sind wesentlich spezifischer für die Zöliakie, jedoch besitzen ca. $2 \%$ der Zöliakie-Patienten einen angeborenen IgA-Mangel, der zu falsch negativen Befunden führen würde. Für eine sichere Diagnostik der Zöliakie ist daher die kombinierte Analytik von IgG- und IgA-Antigliadin-Antikörpern erforderlich. Hierdurch erlangt man eine Sensitivität von $96 \%$ bis $100 \%$ bei einer Spezifität von $96 \%$ bis $97 \%$ [41]. Um einen weiteren Vergleich zu den Sensor- Ergebnissen zu bekommen, wurden die Seren auch mit einem kommerziellen Testsystem (IMTEC-Gastro-LIA, Imtec, Berlin) analysiert.

Vor der EIS-Analyse wurden die verschieden Seren analog zu den ELISA-Experimenten verdünnt, um $\mathrm{R}_{\mathrm{ct}}{ }^{-}$ Werte zu erhalten, die innerhalb des linearen Teils der Kalibrationskurve liegen. Wie zu erwarten war, resultierte die Analyse der Seren in höheren IgG Konzentrationen und niedrigeren IgA-Konzentrationen an AntigliadinAntikörpern. Beide Typen von Immunglobulinen besitzen aber mikromolare Konzentrationen (Tab. 2). Die physiologische Konzentration dieser Antikörper im gesunden Menschen liegt bei ca. $90 \mu \mathrm{M}$ für IgG- und bei ca. $22 \mu \mathrm{M}$ für IgA-Antigliadin-Antikörper [42]. Aus der 
Tabelle wird also deutlich, dass die IgG-Werte für die Seren N2 und N3 nur ca. 50\% der Literaturangabe betragen, während N1 mit $210 \mu \mathrm{M}$ einen vielfach höheren Wert besitzt. Die Konzentrationen für den IgA-Antigliadin-Antikörper in den Seren N1 und N3 ist wiederum dreifach höher als in der Literatur angegeben. Hierbei ist aber zu berücksichtigen, dass die Kalibration nur als eine Annäherung betrachtet werden kann, da keine kommerziellen IgA-Antigliadin-Antikörper verfügbar waren. Für die Konzentrationsbestimmung musste also auf die IgG-Kalibrationskurve zurückgegriffen werden. Trotz ähnlicher Molekulargewichte der Immunglobuline vom Typ IgG und IgA kommt es im Fall der IgA-Moleküle zur Aggregatbildungen, was die Bestimmung des $\mathrm{R}_{\mathrm{ct}}$-Wertes stark beeinflussen kann [43]. Weiterhin ist zu berücksichtigen, dass für eine genaue Konzentrationsbestimmung der Autoantikörper Kalibrationskurven mit humanen Antigliadin-Antikörpern hätten etabliert werden müssen. Gereinigte humane Antigliadin-Antikörper-Lösungen mit Konzentrationsangaben sind jedoch bisher weder für den IgG- noch den IgA-Typ erhältlich.

\begin{tabular}{|l|l|l|l|r|l|l|}
\hline Serum & $\begin{array}{l}\text { Verdün- } \\
\text { nung }\end{array}$ & $\begin{array}{l}\text { Anti- } \\
\text { körper } \\
\text { Typ }\end{array}$ & $\begin{array}{l}\text { Rct } \\
(\mathrm{MOhm})\end{array}$ & $\begin{array}{l}\text { Antikörper } \\
\text { Konz. }(\mu \mathrm{M})\end{array}$ & $\begin{array}{l}\text { Seramun } \\
\text { ELISA }\end{array}$ & $\begin{array}{l}\text { Imtec- } \\
\text { Gastro- } \\
\text { LIA }\end{array}$ \\
\hline \multirow{2}{*}{ P1 } & $1: 400$ & $\lg \mathrm{G}$ & 1.38 & $+(840)$ & + & + \\
\cline { 2 - 8 } & $1: 200$ & $\lg \mathrm{A}$ & 1.00 & $+(140)$ & + & + \\
\hline \multirow{2}{*}{ P2 } & $1: 200$ & $\lg \mathrm{G}$ & 1.36 & $+(380)$ & + & - \\
\cline { 2 - 8 } & $1: 200$ & $\lg \mathrm{A}$ & 0.73 & $-(70)$ & - & - \\
\hline \multirow{2}{*}{ N1 } & $1: 100$ & $\lg \mathrm{G}$ & 1.38 & $-(210)$ & - & - \\
\cline { 2 - 8 } & $1: 200$ & $\lg \mathrm{A}$ & 0.67 & $-(60)$ & - & - \\
\hline \multirow{2}{*}{ N2 } & $1: 200$ & $\lg \mathrm{G}$ & 0.56 & $-(40)$ & - & - \\
\cline { 2 - 8 } & $1: 200$ & $\lg \mathrm{A}$ & 0.76 & $-(80)$ & - & - \\
\hline \multirow{2}{*}{ N3 } & $1: 200$ & $\lg \mathrm{C}$ & 0.55 & $-(41)$ & - & - \\
\cline { 2 - 7 } & $1: 200$ & $\lg \mathrm{A}$ & 0.70 & $-(66)$ & - & - \\
\hline
\end{tabular}

Tab 2: Ladungstransferwiderstände $\left(R_{c t}\right.$-Werte) und Antigliadin Antikörperkonzentrationen, die mit dem Biosensor für die verschiedenen Seren bestimmt werden konnten. Zum Vergleich sind auch die Ergebnisse der Analysen mit dem ELISA- und dem IMTECTest gezeigt.

Auch wenn gesicherte Antikörperkonzentrationen für die einzelnen Seren hier nicht angegeben werden können, kann doch mit diesem Biosensor eindeutig zwischen positiven und negativen Seren unterschieden werden. Das negative Serum N1 besitzt zwar im Vergleich zu N2 und N3 einen erhöhten IgG-Wert, trotzdem liegt dieser Wert noch unter den Werten für P1 und P2. In ELISA-Experimenten wurde dieses Serum zwar eindeutig als negativ bewertet, doch hängt dies stark davon ab, bei welcher Signalstärke der cut-off zwischen positiv und negativ gesetzt wird. Der erhöhte IgG-Level $(210 \mu \mathrm{M})$ kann auch von unterschiedlichen Verdünnungsfaktoren herrühren. Die Verdünnung von komplexen Mischungen, wie humanen Seren, ist aufgrund von Ausdünnungseffekten störender Substanzen nicht nur für den Biosensor, sondern auch in ELISA-Analysen ein kritischer Punkt. Betrachtet man die Konzentrationen der AntigliadinAntikörper vom IgA-Typ, so fällt auf, dass nur das Serum P1 eine signifikante Erhöhung aufweist, wogegen die Konzentrationen in P2, N1, N2 und N3 sehr ähnlich sind. Dies stimmt mit den ELISA-Ergebnissen überein.
Hier wird das Serum P2 als negativ hinsichtlich der IgAAntikörper eingestuft. Ein Grund hierfür könnte der schon erwähnte physiologische IgA-Mangel sein, der zu falsch negativen Ergebnissen führen kann.

Die Analyse der Seren mit einem kommerziell erhältlichen Test (IMTEC) zeigte für P1 einen positiven Befund hinsichtlich der IgG- als auch der IgA-Antikörper, wogegen P2 fälschlicherweise als negativ eingestuft wurde. Negativ-Seren wurden richtigerweise auch als negativ erkannt. Im Vergleich zum entwickelten Biosensor und dem ELISA erlaubt der IMTEC-Test nur eine Ja-/ Nein-Antwort und gibt keine Konzentrationsangaben. Zusammenfassend lässt sich festhalten, dass der entwickelte Biosensor eine zuverlässige Analytik für die Konzentrationsbestimmung von Antigliadin-Antikörpern in humanen Seren bietet.

\section{$4 \quad$ Zusammenfassung}

In dieser Arbeit wurde ein Biosensor für die Analytik von Antigliadin-Antikörpern im humanen Serum entwickelt. Der Biosensor basiert auf Goldelektroden, die mit dem Polyelektrolyten PSS oder mit 3-Mercaptopropionsäure modifiziert worden waren, um darauf das Antigen Gliadin zu koppeln. Im Anschluss an die Inkubation des Biosensors mit der Probe wurde zur Verstärkung des Antikörper-Antigen-Signals ein zweiter Inkubationsschritt mit einem POD-markierten Antikörper und anschließender POD- katalysierter Oxidation von AEC durchgeführt. Der Zuwachs der Schichtdicke auf der Elektrodenoberfläche im Verlauf des Sandwich-Assays führte zu einer Zunahme in der Impedanz der Elektrode. Diese Zunahme wurde durch EIS in Anwesenheit von Ferri-/Ferrocyanide gemessen. Hierbei konnte der Ladungstransferwiderstand $\left(\mathrm{R}_{\mathrm{ct}}\right)$ als Sensorparameter identifiziert werden. Es wurde eine Kalibrationskurve für Antigliadin-Antikörper etabliert, in dem eine Korrelation zwischen $\mathrm{R}_{\mathrm{ct}}$ Werten und verschiedenen Antikörperkonzentrationen aufgestellt wurde. Mit Hilfe dieser Eichkurve konnte die Konzentration von Antigliadin-Antikörpern in verschiedenen humanen Seren bestimmt werden.

\section{Danksagung}

Diese Arbeit wurde durch das Bundesministerium für Bildung und Forschung (BMBF) (Projekt 03I1315C) gefördert. Die Autoren bedanken sich bei der Seramun Diagnostica $\mathrm{GmbH}$ für die freundliche und intensive Kooperation im Rahmen des Projektes. 


\section{Referenzen}

[1] Weiss, W./Vogelmeier, C./A. Görg, Electrophoresis 1993, 14, 805

[2] Navratilova, I./Skaldal, P., Bioelectrochemistry 2004, 62,11

[3] Cooreman, P./Thoelen, R./Manca, J./van de Ven, M./ Vermeeren, V./Michiels, I./Ameloot, M./Wagner, P., Biosensors and Bioelctronics 2005, 20, 2151

[4] Tang, D./Yuan, R./Chai, Y./Fu, Y./Dai, J./Liu, Y./Zhong, X., Biosensors and Bioelectronics 2005, 21, 539

[5] Zhang, L./Yuan, R./Huang, X./Chai, Y./Tang, D./Cao, S., Anal. Bioanal. Chem. 2005, 381, 1036

[6] Ding, Y./Wang, H./Shen, G./Ruqin, Y., Anal. Bioanal. Chem. 2005, 382, 1491-1499

[7] Gonzáles-Martinez, M. A./Puchades, R./Maquieira, A., Trends Anal. Chem. 1999, 18, 204

[8] van Emon, J. M./Lopez-Avila, V., Anal. Chem. 1992, 64, $79 a$

[9] Märtlbauer, E./Usleber, E./Schneider, E./Dietrich, R., Analyst 1994, 119, 2543

[10] Warsinke, A./Benkert, A./Scheller, F. W., Fr. J. Anal. Chem. 2000, 366, 622

[11] Skladal, P., Electroanalysis 1997, 9, 737

[12] Cousino, M. A./Heinemann, W. R./Halsall, H. B., Ann. Chim. 1997, 87, 93

[13] Fernández-Sánchez, C./Gonzáles-García, M. B./CostaGarcía, A., Biosens. Bioelectron. 2000, 14, 917

[14] Schreiber, A./Feldbrugge, R./Key, G./Glatz, J. F. C./Spencer, F., Biosens. Bioelectron. 1997, 12, 1131

[15] Niwi, O./Xu, Y./Halsall, H. B./Heineman, W. R., Anal. Chem. 1993, 65, 1559

[16] Sadik, O. A./van Emon, J. M., Biosens. Bioelectron. 1996, 11, i

[17] Wang, M./Wang, L./Wang, G./Ji, X./Bai, Y./Li, T./Gong, S./Li, J., Biosensors and Bioelectronics 2004, 19, 575

[18] Łoś, M./Łoś, J. M./Blohm, L./Spiller, E./Grunwald, T./ Albers, J./Hintsche, R./Wegrzyn, G., Lett Appl Microbiol 2005, 40, 479

[19] Rubinstein, I., Physical Electrochemisty 1995, Marcel Dekker Inc. New York, 243

[20] Knoll, W./Frank, C. W./Heibel, C./Naumann, R./Offenhäusser, A./Rühe, J./Schmidt, E. K./Shen, W. W./Sinner, A., Reviews in Molecular Biotechnology 2000, 74, 137

[21] Darain, F./Park, D./Park, J./Shim, Y., Biosensors and Bioelectronics 2004, 19, 1245

[22] Wang, J./Pamidi, P. V., Anal. Chem. 1998, 70, 1171

[23] Lee, H. Y./Park, J. W., T. Kawai, Electroanalysis 2004, 16, 1999

[24] Ouerghi, O./Touhami, A./Jaffrezic-Renault, N./Martelet, C./Ben Ouada, H./Cosnier, S., Bioelectrochemistry $2002,56,131$

[25] Mirsky, V. M./Riepl, M./Wolfbeis, O. S., Biosensors \& Bioelectronics 1997, 12, 977
[26] Riepl, M./Mirsky, V. M./Novotny, I./Tvarozek, V./Rehacek, V./Wolfbeis, O. S., Anal. Chim. Acta 1999, 392, 77

[27] Willner, I./Katz, E., Electroanalysis 2003, 15, 913

[28] Campuzano, S./Galvez, R./Pedrero, M./Manuel de Villana, F. J./Pingarron, J. M., Anal Bioanal Chem. 2003, 377,600

[29] Etienne, O./Picart, C./Taddei, C./Haikel, Y./Dimarcq, J. L./Schaaf, P./Voegel, J. C./Ogier, J. A./Egles, C., Agents and Chemotherapy 2004, Okt., 3662

[30] Beissenhirtz, M. K./Scheller, F. W./Lisdat, F., Anal. Chem. 2004, 76, 4665

[31] Ladam, G./Schaaf, P./Cuisinier, F. J. G./Dechner, G./ Voegel, J.-C., Langmuir 2001, 17(3), 878

[32] Caruso, F./Furlong, D. N./Ariga, K./Ichinose, I./Kunitake, T., Langmuir, 1998, 14 (16), 4549

[33] Gergely, C./Bahi, S./Szalontai, B./Flores, H./Schaaf, P./ Voegel, J.-C./Cuisinier, F. J. G., Langmuir, 2004, 20 (13), 5575

[34] Muratsugu, M./Ohta, F./Miya, Y./Hosokawa, T./Kurosawa, S., N. Kamo, H. Ikeda, Anal. Chem. 1993, 65, 2933

[35] Bradford, M., Anal. Biochem., 1976, 72, 248

[36] Sauerbrey, G., Z. Phys. 1959, 155, 206

[37] Cavic, B. A./Thompson, M., Analyst, 1998, 123, 2191

[38] Lyle, E.-L./Hayward, L., M. Thomson, Analyst, 2002, 127,1596

[39] Parsons, R./Electroanal, J., Chem. 1998, 452, 1

[40] Hock, B./Nießner, R., VCH Verlagsgesellschaft, Weinheim, DFG research report 1995

[41] Semrad, C. E., Celiac Disease and Gluten Sensitivity 2000 (www.gflinks.com)

[42] Janeway, C. A./Trevers, P./Walport, M., Shlomchik Immunologie, 5. Auflage 2002, Spektrum Verlag GmbH, Heidelberg

[43] Lottspeich, F., Bioanalytik, Spectrum Verlag, 1998, 70

\section{Literatur}

Beissenhirtz, M. K./Scheller, F. W./Lisdat, F., Anal. Chem. $2004,76,4665$

Bradford, M., Anal. Biochem., 1976, 72, 248

Campuzano, S./Galvez, R./Pedrero, M./Manuel de Villana, F. J./Pingarron, J. M., Anal Bioanal Chem. 2003, 377, 600

Caruso, F./Furlong, D. N./Ariga, K./Ichinose, I./Kunitake, T., Langmuir, 1998, 14 (16), 4549

Cavic, B. A./Thompson, M., Analyst, 1998, 123, 2191

Cooreman, P./Thoelen, R./Manca, J./van de Ven, M./Vermeeren, V./Michiels, I./Ameloot, M./Wagner, P., Biosensors and Bioelctronics 2005, 20, 2151

Cousino, M. A./Heinemann, W. R./Halsall, H. B., Ann. Chim. 1997, 87, 93 
Darain, F./Park, D./Park, J./Shim, Y., Biosensors and Bioelectronics 2004, 19, 1245

Ding, Y./Wang, H./Shen, G./Ruqin, Y., Anal. Bioanal. Chem. 2005, 382, 1491-1499

van Emon, J. M./Lopez-Avila, V., Anal. Chem. 1992, 64, $79 \mathrm{a}$

Etienne, O./Picart, C./Taddei, C./Haikel, Y./Dimarcq, J. L./ Schaaf, P./Voegel, J. C./Ogier, J. A./Egles, C., Agents and Chemotherapy 2004, Okt., 3662

Fernández-Sánchez, C./Gonzáles-García, M. B./Costa-García, A., Biosens. Bioelectron. 2000, 14, 917

Gergely, C./Bahi, S./Szalontai, B./Flores, H./Schaaf, P./Voegel, J.-C./Cuisinier, F. J. G., Langmuir, 2004, 20 (13), 5575

Gonzáles-Martinez, M. A./Puchades, R./Maquieira, A., Trends Anal. Chem. 1999, 18, 204

Hock, B./Nießner, R., VCH Verlagsgesellschaft, Weinheim, DFG research report 1995

Janeway, C. A./Trevers, P./Walport, M./Shlomchik, M., Immunologie, 5. Auflage 2002, Spektrum Verlag GmbH, Heidelberg

Knoll, W./Frank, C. W./Heibel, C./Naumann, R./Offenhäusser, A./Rühe, J./Schmidt, E. K./Shen, W. W./Sinner, A., Reviews in Molecular Biotechnology 2000, 74, 137

Ladam, G./Schaaf, P./Cuisinier, F. J. G./Dechner, G./Voegel, J.-C., Langmuir 2001, 17(3), 878

Lee, H. Y./Park, J. W./Kawai, T., Electroanalysis 2004, 16, 1999

Łoś, M./Łoś, J. M./Blohm, L./Spiller, E./Grunwald, T./Albers, J./Hintsche, R./Wegrzyn, G., Lett Appl Microbiol 2005, 40,479

Lottspeich, F., Bioanalytik, Spectrum Verlag, 1998, 70

Lyle, E.-L./Hayward, L./Thomson, M., Analyst, 2002, 127, 1596

Märtlbauer, E./Usleber, E./Schneider, E., Dietrich, R., Analyst 1994, 119, 2543

Mirsky, V. M./Riepl, M., Wolfbeis, O. S., Biosensors \& Bioelectronics 1997, 12, 977

Muratsugu, M./Ohta, F./Miya, Y./Hosokawa, T./Kurosawa, S./Kamo, N./Ikeda, H., Anal. Chem. 1993, 65, 2933

Navratilova, I., Skaldal, P., Bioelectrochemistry 2004, 62, 11

Niwi, O./Xu, Y./Halsall, H. B./Heineman, W. R., Anal. Chem. 1993, 65, 1559

Ouerghi, O./Touhami, A./Jaffrezic-Renault, N./Martelet, C./ Ben Ouada, H./Cosnier, S., Bioelectrochemistry 2002, 56,131

Parsons, R., J. Electroanal. Chem. 1998, 452, 1

Riepl, M./Mirsky, V. M./Novotny, I., Tvarozek, V./Rehacek, V./Wolfbeis, O. S., Anal. Chim. Acta 1999, 392, 77

Rubinstein, I., Physical Electrochemisty 1995, Marcel Dekker Inc. New York, 243

Sadik, O. A./van Emon, J. M., Biosens. Bioelectron. 1996, $11, \mathrm{i}$

Sauerbrey, G., Z. Phys. 1959, 155, 206
Schreiber, A./Feldbrugge, R./Key, G./Glatz, J. F. C./Spencer, F., Biosens. Bioelectron. 1997, 12, 1131

Semrad, C. E., Celiac Disease and Gluten Sensitivity 2000, (www.gflinks.com)

Skladal, P., Electroanalysis 1997, 9, 737

Tang, D./Yuan, R./Chai, Y./Fu, Y./Dai, J./Liu, Y./Zhong, X., Biosensors and Bioelectronics 2005, 21, 539

Wang, J./Pamidi, P. V., Anal. Chem. 1998, 70, 1171

Wang, M./Wang, L./Wang, G./Ji, X./Bai, Y./Li, T./Gong, S./ Li, J., Biosensors and Bioelectronics 2004, 19, 575

Warsinke, A./Benkert, A./Scheller, F. W., Fr. J. Anal. Chem. $2000,366,622$

Weiss, W./Vogelmeier, C./Görg, A., Electrophoresis 1993, 14,805

Willner, I., Katz, E., Electroanalysis 2003, 15, 913

Zhang, L./Yuan, R./Huang, X./Chai, Y./Tang, D./Cao, S., Anal. Bioanal. Chem. 2005, 381, 1036

\section{Autoren}

\section{Thomas Balkenhohl}

\section{Falco Beutler}

Dipl.-Ing. (FH) Daniel Schäfer

\section{Prof. Dr. Fred Lisdat}

Technische Fachhochschule Wildau

Biosystemtechnik

Tel. +49 3375 508-134

fred.lisdat@tfh-wildau.de 